\section{WEB WATCH}

Integrity in science

Corporate sponsorship of scientific research is a controversial issue. Should, for example, a company that makes a certain product then fund research into the effects of that product? Conversely, would such funding leave researchers open to claims of bias?

As research institutes, publishers and editorial boards draw up policies on the disclosure of financial interests, the Center for Science in the Public Interest (CSPI), a consumer-advocacy group based in Washington DC, has launched an online database that lists the links of scientists and non-profit organizations with industry.

The database is part of the 'Integrity in Science' project, which is "concerned about the link between industry and science and how the demands of the former can undermine the public-interest mission of the latter". The scientists and organizations listed come mainly from the fields of nutrition, the environment, toxicology and medicine

Information on the database has been culled from various sources, including scholarly publications, news articles, résumés, industry bulletins, web sites and conference programmes. There are two points of entry $-\mathrm{a}$ searchable database of scientists, and a list of the non-profit organizations with links to industry.

The site is simple, attractive and easy to navigate, with additional links to 'resources', which include online articles, a bibliography, and links to conflict-of-interest guidelines and selected laws and legislative materials. A further set of links provide access to the web sites of scientists and organizations with an interest in the ties between research and industry. And the 'actions and articles' page contains letters and articles on the subject, updated regularly over recent months.

Alison Mitchell

\section{A glance upstream}

Lipid rafts are dynamic assemblies of cholesterol and sphingolipids, which form microenvironments in the lipid bilayer. Raft clustering is proposed to mediate the activation of signalling cascades, perhaps by bringing together different components of the pathway. But what triggers clustering? Reporting in Science, Khan and colleagues now show that, during activation of $\mathrm{T}$ cells, a factor that mediates the formation of the immunological synapse is the same protein as that which mediates formation of neuromuscular junctions - agrin. Khan and colleagues find that, upon activation of primary immune cells, agrin becomes

\title{
CELL DIVISION
}

\section{Division of labour}

Centrosomes - the main microtubule organizing centres of the cell - make sure that, upon division, each cell receives an equal number of chromosomes. To this end, centrosomes duplicate once per cell cycle, separate, and then nucleate the two end points of the mitotic spindle. The cell is thought to use two types of regulator to ensure that this crucial duplication takes place: first, cell-cycle factors, which regulate when centrosome duplication takes place; and second, intrinsic factors, which ensure only one round of duplication occurs per cell cycle. Now, reporting in Cell, O'Connell and colleagues show that ZYG-1, a protein kinase that localizes to centrosomes, fufils the second criterion - it mediates duplication but is not required for cell-cycle progression. They also highlight a division of labour between paternal and maternal ZYG-1 - a regulatory mechanism that ensures coordination between the gametes during fertilization.

The $z y g-1$ gene was previously identified in Caenorhabditis elegans as being essential for cell division. In its absence, cells fail to form a bipolar spindle but instead produce a monopolar spindle. This could occur either because the centrosomes (which consist of a pair of centrioles) have not reproduced properly or because they cannot separate. Looking more closely, O'Connell and colleagues were surprised to find that the monopolar spindles contained a single unpaired centriole - a finding that was not expected in either model. This led them to conclude that ZYG-1 is required for centriole formation.

During fertilization, a pair of centrioles is usually provided by the sperm, indicating that the defect might be due to a paternal effect. Using genetics, the authors asked what happens when only the paternal copy of $z y g-1$ is lost. They found that the first cell division was blocked owing to the absence of a bipolar spindle. But, surprisingly, during the following divisions, a normal spindle formed and cell division proceeded. Extrapolating from this, O'Connell and colleagues proposed that maternal $z y g-1$ takes over after the first round of division - a prediction that they then confirmed by temperature-shift experiments.

Still intrigued by the presence of a single unpaired centriole in $z y g-1$ mutant zygotes, the authors next asked when paternal $z y g-1$ activity is required, before or after fertilization. They found that paternal ZYG1 is required before fertilization, during male meiosis, for the synthesis of a second centriole.

Cloning of $z y g-1$ revealed it to be a unique protein kinase that does colocalized with the T-cell receptor (TCR) on the cell surface. As neural agrin can induce aggregation of acetylcholine receptors on muscle cells, the authors wondered whether expression of agrin in activated lymphocytes can induce aggregation of TCRs. Treating cells with agrin purified from activated cells (agrin ${ }_{\text {act }}$ ), they showed, triggered lipid raft clustering. Furthermore, agrin decreases proliferation thresholds - a measure of lymphocyte

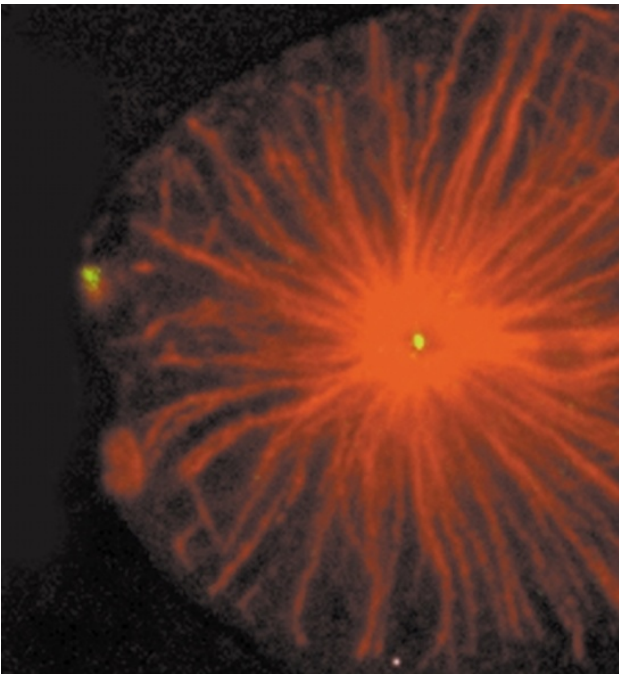

The figure shows a one-cell embryo, stained for ZYG-1 (green) and $\beta$-tubulin (red). ZYG-1 localizes to the poles. (C) (2001) Elsevier Science.

not show homology to any specific protein kinase family. The authors also could not tell whether it is a serine or a tyrosine kinase. So is the kinase domain functional? To test this, they compared wild-type and kinase-dead versions of ZYG-1 in vitro. Although they could not find other substrates, they showed that ZYG-1 undergoes auto-phosphorylation. Finally, molecular complementation experiments indicated that the kinase activity is required for rescue of the centrosome duplication defect.

Does the expression pattern of zyg-1 support a role in centrosome duplication? To address this question, O'Connell and colleagues raised an antibody against ZYG-1 and showed that it associates almost exclusively at the centrosomes at the 